Research Paper

\title{
Sex-specific Association of the Zinc Finger Protein 259 rs2075290 Polymorphism and Serum Lipid Levels
}

\author{
Lynn Htet Htet Aung ${ }^{1}$, Rui-Xing Yin ${ }^{1}{ }^{凶}$, Dong-Feng Wu ${ }^{1}$, Wei Wang ${ }^{1}$, Jin-Zhen Wu ${ }^{1}$, Cheng-Wu Liu ${ }^{2}$ \\ 1. Department of Cardiology, Institute of Cardiovascular Diseases, the First Affiliated Hospital, Guangxi Medical University, Nanning, \\ Guangxi, People's Republic of China; \\ 2. Department of Pathophysiology, School of Premedical Sciences, Guangxi Medical University, Nanning, Guangxi, People's Republic of \\ China.
}

\begin{abstract}
$\triangle$ Corresponding author: Dr. Rui-Xing Yin, Department of Cardiology, Institute of Cardiovascular Diseases, the First Affiliated Hospital, Guangxi Medical University, 22 Shuangyong Road, Nanning 530021, Guangxi, People's Republic of China; Tel: +86-771-5326125; Fax: +86-771-5353342; E-mail: yinruixing@163.com.
\end{abstract}

(C) Ivyspring International Publisher. This is an open-access article distributed under the terms of the Creative Commons License (http://creativecommons.org/ licenses/by-nc-nd/3.0/). Reproduction is permitted for personal, noncommercial use, provided that the article is in whole, unmodified, and properly cited.

Received: 2014.0I.04; Accepted: 2014.02.27; Published: 2014.03.16

\begin{abstract}
Background: Little is known about the association of ZNF259 rs2075290 single nucleotide polymorphism (SNP) and serum lipid levels in the Chinese population. This study aimed to detect the association of ZNF259 rs2075290 SNP and environmental factors with serum lipid levels between males and females in the Mulao and Han populations.

Methods and Results: Genotyping of ZNF259 rs2075290 SNP was performed in 788 of Mulao and 778 of Han participants using polymerase chain reaction and restriction fragment length polymorphism. The genotype frequencies were significantly different between Mulao and Han populations (AA, 50.1\% Vs 58.9\%; AG, 42.3\% Vs 35.7\%; GG, 7.6\% Vs 5.4\%, $P=0.002$ ) and between Han males and females (AA, 64.5\% Vs 55.2\%; AG, 28.3\% Vs 40.6\%; GG, 7.2\% Vs 4.2\%, $P=0.001$ ). Serum levels of triglyceride (TG) in Mulao males, and total cholesterol (TC), TG and low-density lipoprotein cholesterol (LDL-C) in Mulao females were different between the AA and AG/GG genotypes $(P<0.05-0.001)$. Serum TC, LDL-C and apolipoprotein (Apo) AI levels in Han males, and TG and $A p o B$ levels and $A p o A I / A p o B$ ratio in Han females were different between the $A A$ and AG/GG genotypes $(P<0.05-0.00 I)$. An interaction between ZNF259 rs2075290 polymorphism and male gender on serum TC, LDL-C, and ApoAl levels was noted in Han population $(P<$ 0.05-0.01) but not in Mulao's.

Conclusions: The subjects with AG/GG genotype in Mulao males and females and Han females have less favorable lipid profiles than those with AA genotype. In contrast, the subjects with AG/GG genotype in Han males have more favorable lipid profiles than those with AA genotype. These findings suggest that the association between ZNF259 rs2075290 SNP and serum lipid levels might have ethnic- and/or sex-specificity.
\end{abstract}

Key words: lipids; sex-specific association; zinc finger protein 259 (ZNF259); single nucleotide polymorphism; environmental factors.

\section{Introduction}

Both dyslipidemia and sex are associated with the risk of cardiovascular diseases (CVDs). The striking differences in lipid and lipoprotein metabolism between men and women bring a significant issue to the management and prognosis of CVD [1, 2]. The relative risk of CVD in subjects with high plasma triglyceride (TG) levels is higher in women than in men. Moreover, changes of TG or high-density lipoprotein cholesterol (HDL-C) in women are better predicators of CVD risk than those of total cholesterol (TC) or 
low-density lipoprotein cholesterol (LDL-C) [3]. It has also reported that regulating serum TG and HDL-C level is more essential in women than in men [4]. However, the reason for these differences concerned with gender is not yet fully understood. It may be due to 1) certain genes which are expressed in sexually dimorphic manner; 2) sexual hormones which determine phenotypic variations; and/or 3) difference in environmental factors $[1,2,5]$. Therefore, the understanding of sex-specific association between SNPs and serum lipid levels is crucial for improving lipid control in personalized medicine.

Recently, several genome wide association studies (GWAS) have reported the association of many single nucleotide polymorphisms (SNPs) near the zinc finger protein 259 gene (ZNF259; also known as zinc finger protein ZPR1; Gene ID: 8882; OMIM: 603901; located in chromosome 11q23.3) with one or more lipid traits [6-9] though the biological function of ZPR1 in lipid metabolism is unknown. So far, the well-known function of ZPR1 is that it is essential for normal functioning of the nucleoli during cell proliferation [10,11]. A sex-stratified meta-analysis of lipid-related gene variants in European Americans has shown that rs28927680 and rs3135506 SNPs near the ZNF259 gene region had a sex specific effect on fasting TG levels. This effect was replicable in Mexican Americans/Hispanics [12]. Another meta-analysis of GWAS on metabolic syndrome (MetS) has identified the rs2075290 SNP in the intronic region of ZNF259 as TG-related loci in European populations [13]. However, in Chinese population, whether ZNF259 rs2075290 SNP is associated with serum lipid levels or whether it exhibits sex specific association like the previously reported ZNF259 SNPs remains elusive.

Among 56 ethnic groups in China, Han is the largest one. Mulao, on the other hand, is one of the minorities with a population of 207,352 according to the China's fifth national census in 2000. Approximately ninety percent of Mulao peoples are dwelling in the Luocheng Mulao Autonomous County, Guangxi Zhuang Autonomous Region. "Ling", "Jin" and "Bendiren" are local alternative names for Mulao ethnic group. Historical data trace the history of this ethnic minority back to the Jin Dynasty (AD 265-420). Mulao nationality is a relatively conservative and isolated minority, and preserves their custom of intra-ethnic marriage. Interestingly, they have their culture of consanguineous marriage to cousins of maternal side, suggesting that the genetic background of Mulao population may be less heterogeneous within the population. The Mulao peoples are the descendants of the ancient "Baiyue tribe" in southern China and ethnically related to the neighboring ethnic groups. The recent molecular anthropological data showed that Mulao has much closer genetic relationship with the other minorities in Guangxi than with the Han nationality [14]. In addition, several previous studies have revealed that the associations of variants in several lipid-related genes and serum lipid levels are significantly different between the Mulao and Han populations and their gender subgroups [15-17]. This study, therefore, was undertaken to detect the association of ZNF259 rs2075290 SNP and several environmental factors with serum lipid levels between males and females in the Mulao and Han populations.

\section{Materials and Methods}

\section{Subjects}

Two groups of study population including 788 unrelated participants (346 males, $43.9 \%$ and 442 females, $56.1 \%$ ) of Mulao and 778 unrelated subjects (307 males, 39.5\% and 471 females, 60.5\%) of Han were randomly selected from our previous stratified randomized samples [18, 19]. All participants were agricultural workers from Luocheng Mulao Autonomous County, Guangxi Zhuang Autonomous Region, People's Republic of China. The participants' age ranged from 15 to 80 years with the mean age of $48.79 \pm 15.93$ years in Mulao and $49.33 \pm 16.19$ years in Han; respectively. The age distribution and gender ratio were matched between the two groups. All participants were essentially healthy with no history of CVD such as coronary artery disease (CAD) and stroke, diabetes, hyper- or hypo-thyroids, and chronic renal disease. They were free from medications known to affect serum lipid levels. Informed consent was taken from all participants. The study design was approved by the Ethics Committee of the First Affiliated Hospital, Guangxi Medical University.

\section{Epidemiological survey}

The epidemiological survey was carried out using internationally standardized methods, following a common protocol [18, 19]. Information on demographics, socioeconomic status, and lifestyle factors was collected with standardized questionnaires. Alcohol consumption was categorized into groups of grams of alcohol per day: $\leq 25$ and $>25$. Smoking status was categorized into groups of cigarettes per day: $\leq 20$ and $>20$. Several parameters such as blood pressure, height, weight, waist circumference, and body mass index (BMI) were measured. The methods of measuring above parameters were referred to previous studies [15, 17].

\section{Biochemical measurements}

A fasting venous blood sample of $5 \mathrm{ml}$ was drawn from the participants. The levels of TC, TG, HDL-C and LDL-C in the samples were determined 
by enzymatic methods with commercially available kits. Serum apolipoprotein (Apo) A1 and ApoB levels were assessed by the immuneturbidimetric immunoassay $[18,19]$.

\section{Genotyping}

Genomic DNA was isolated from peripheral blood leukocytes using the phenol-chloroform method [15, 17]. The ZNF259 rs2075290 SNP was genotyped by polymerase chain reaction and restriction fragment length polymorphism (PCR-RFLP). PCR amplification was performed using 5'-GAGTCTATGGGATAATGTGC-3' as the forward and 5'-AATTCCAGGCCTAACTTTGG-3' as reversed primer pair. Each amplification reaction was performed in a total volume of $25 \mathrm{ml}, 12.5 \mu \mathrm{l}$ of $2 \times$ Taq PCR MasterMix (constituent: $0.1 \mathrm{U}$ Taq polymerase $/ \mu \mathrm{l}, 500 \mu \mathrm{M}$ dNTP each and PCR buffer) and nuclease-free water $8.5 \mu \mathrm{l}, 20 \mathrm{pmol} / \mathrm{L}$ of each primer and $100 \mathrm{ng}$ of genomic DNA, processing started with 5 min of pre-denaturing at $95^{\circ} \mathrm{C}$ and followed by $45 \mathrm{~s}$ of denaturing at $94^{\circ} \mathrm{C}, 30 \mathrm{~s}$ of annealing at $56.7^{\circ} \mathrm{C}$ and 1 min of elongation at $72{ }^{\circ} \mathrm{C}$ for 35 cycles. The amplification was completed by a final extension at $72{ }^{\circ} \mathrm{C}$ for $10 \mathrm{~min}$. Then each restriction enzyme reaction was performed with $10 \mu \mathrm{l}$ of amplified DNA, $8 \mu \mathrm{l}$ of nuclease-free water, $1 \mu \mathrm{l}$ of $10 \times$ buffer solution, and $10 \mathrm{U}$ of $S s p$ I enzyme in a total volume of $20 \mu \mathrm{l}$ digested at $37^{\circ} \mathrm{C}$ overnight. After restriction enzyme digestion of the amplified DNA, the digestive products were separated by electrophoresis on $2 \%$ agrose gel. The length of each digested DNA fragment was determined by comparing migration of a sample with that of standard DNA marker. Genotypes were scored by an experienced reader blinded to the epidemiological and lipid results. Six samples (each genotype in two; respectively) detected by the PCR-RFLP were also confirmed by direct sequencing. The PCR products were purified by low melting point gel electrophoresis and phenol extraction, and then the DNA sequences were analyzed using an ABI Prism 3100 (Applied Biosystems) in Shanghai Sangon Biological Engineering Technology \& Services Co., Ltd., People's Republic of China.

\section{Diagnostic criteria}

The normal values of serum TC, TG, HDL-C, LDL-C, ApoA1 and ApoB levels, and the ratio of ApoA1 to ApoB in our Clinical Science Experiment Center were 3.10-5.17, 0.56-1.70, 1.16-1.42, 2.70-3.10 $\mathrm{mmol} / \mathrm{L}, 1.20-1.60,0.80-1.05 \mathrm{~g} / \mathrm{L}$, and 1.00-2.50; respectively $[16,20]$.

\section{Statistical analysis}

The statistical analyses were performed with the statistical software package SPSS 17.0 (SPSS Inc., Chicago, Illinois). The quantitative variables were presented as mean \pm standard deviation (serum TG levels were presented as medians and interquartile ranges). Allele frequency was determined via direct counting, and the Hardy-Weinberg equilibrium was verified with the standard goodness-of-fit test. The genotype distribution between the groups was analyzed by the chi-square test. General characteristics between two ethnic groups were compared by the Student's unpaired $t$-test. The association between genotypes and serum lipid parameters was tested by analysis of covariance (ANCOVA). Age, sex, BMI, smoking, and alcohol consumption were adjusted for the statistical analysis. A factorial design covariance analysis was performed to assess the interaction between genotypes and gender after controlling for potential confounders including age, BMI, smoking, and alcohol consumption. Multivariable linear regression analyses with stepwise modeling were used to determine the correlation between genotypes $(\mathrm{AA}=1$, $\mathrm{AG}=2, \mathrm{GG}=3$ ) or alleles (the $\mathrm{G}$ allele non-carrier $=1$, the $G$ allele carrier $=2$ ) and several environmental factors with serum lipid levels in males and females of Mulao and Han populations. Two sided $P$ value $<0.05$ was considered statistically significant.

\section{Results}

\section{General and biochemical characteristics of the subjects}

Table 1 compares the general characteristics and serum lipid profiles between males and females in Mulao and Han ethnic groups. The values of height, weight, waist circumference, BMI, diastolic blood pressure, and the percentages of subjects consuming alcohol were different between men and women in both ethnic groups $(P<0.05-0.001)$. The values of systolic blood pressure and pulse pressure were different between men and women in Han $(P<0.001$ for each) but not in Mulao. Overall, men had higher values of general characteristic parameters than women in both ethnic groups. In Mulao, men had higher serum TG and ApoB levels, and lower HDL-C levels and ApoA1/ApoB ratio than the women $(P<$ 0.05-0.01). In Han, males had higher TC, TG, LDL-C and ApoB levels, and lower ApoA1/ApoB ratio than the females $(P<0.05-0.001)$. 
Table I. Comparison of demographic, lifestyle characteristics and serum lipid levels between males and females of the Mulao and Han populations.

\begin{tabular}{|c|c|c|c|c|}
\hline \multirow[t]{2}{*}{ Parameter } & \multicolumn{2}{|c|}{ Mulao $(\mathrm{n}=788)$} & \multicolumn{2}{|l|}{$\operatorname{Han}(\mathrm{n}=778)$} \\
\hline & Male & Female & Male & Female \\
\hline Number [n (\%)] & $346(43.9)$ & $442(56.1)$ & $307(39.5)$ & $471(60.5)$ \\
\hline Age (years) & $47.35 \pm 15.37$ & $48.76 \pm 15.58$ & $50.17 \pm 17.82$ & $49.32 \pm 15.25$ \\
\hline Height $(\mathrm{cm})$ & $161.78 \pm 5.01$ & $151.06 \pm 6.12 \mathrm{a}$ & $160.20 \pm 8.05$ & $151.22 \pm 5.69 \mathrm{a}$ \\
\hline Weight (kg) & $57.79 \pm 9.10$ & $49.18 \pm 8.10$ a & $58.68 \pm 8.84$ & $49.83 \pm 6.91$ a \\
\hline Body mass index $\left(\mathrm{kg} / \mathrm{m}^{2}\right)$ & $22.04 \pm 3.02$ & $21.53 \pm 3.12 \mathrm{c}$ & $22.96 \pm 3.48$ & $21.80 \pm 2.89$ a \\
\hline Waist circumference $(\mathrm{cm})$ & $77.05 \pm 8.90$ & $73.04 \pm 8.07$ a & $77.54 \pm 7.87$ & $73.29 \pm 7.25^{a}$ \\
\hline Systolic blood pressure (mmHg) & $128.38 \pm 19.78$ & $126.13 \pm 21.39$ & $135.63 \pm 18.86$ & $124.31 \pm 17.30$ a \\
\hline Diastolic blood pressure (mmHg) & $82.31 \pm 11.71$ & $78.63 \pm 10.61$ a & $83.99 \pm 11.18$ & $80.29 \pm 10.79 \mathrm{a}$ \\
\hline Pulse pressure $(\mathrm{mmHg})$ & $46.07 \pm 13.98$ & $47.50 \pm 16.48$ & $51.64 \pm 13.95$ & $44.03 \pm 11.69 \mathrm{a}$ \\
\hline \multicolumn{5}{|l|}{ Cigarette smoking [n (\%)] } \\
\hline Nonsmoker & $204(59.0)$ & $440(99.5)$ & $172(56.0)$ & $465(98.7)$ \\
\hline$\leq 20$ cigarettes/day & $41(11.8)$ & $1(2)$ & $41(13.4)$ & $4(0.8)$ \\
\hline$>20$ cigarettes/day & $101(29.2)$ & $1(2)^{a}$ & $94(30.6)$ & $2(0.4)^{a}$ \\
\hline \multicolumn{5}{|l|}{ Alcohol consumption [n (\%)] } \\
\hline Nondrinker & $203(58.7)$ & 439 (99.3) & $173(56.4)$ & $464(98.5)$ \\
\hline$\leq 25 \mathrm{~g} /$ day & 49 (14.2) & $1(0.2)$ & $62(20.2)$ & $4(0.8)$ \\
\hline$>25 \mathrm{~g} /$ day & $94(27.2)$ & $2(0.5)^{a}$ & $72(23.5)$ & $3(0.6)^{a}$ \\
\hline Blood glucose (mmol/L) & $5.95 \pm 1.82$ & $5.87 \pm 1.40$ & $6.01 \pm 1.78$ & $5.93 \pm 1.36$ \\
\hline Total cholesterol (mmol/L) & $5.01 \pm 1.11$ & $4.88 \pm 1.17$ & $5.10 \pm 0.94$ & $4.83 \pm 1.04$ b \\
\hline Triglyceride (mmol/L) & $1.11(1.03)$ & $1.02(0.66)^{c}$ & $1.14(0.91)$ & $0.97(0.78)^{a}$ \\
\hline HDL-C (mmol/L) & $1.72 \pm 0.56$ & $1.76 \pm 0.42^{c}$ & $1.68 \pm 0.42$ & $1.78 \pm 0.61$ \\
\hline LDL-C (mmol/L) & $2.92 \pm 0.85$ & $2.91 \pm 0.87$ & $2.95 \pm 0.79$ & $2.82 \pm 0.87 c$ \\
\hline Apolipoprotein (Apo) A1 (g/L) & $1.31 \pm 0.42$ & $1.30 \pm 0.40$ & $1.35 \pm 0.28$ & $1.32 \pm 0.26$ \\
\hline ApoB (g/L) & $1.04 \pm 0.66$ & $0.91 \pm 0.47 \mathrm{~b}$ & $0.90 \pm 0.20$ & $0.81 \pm 0.19$ a \\
\hline ApoA1/ApoB & $1.54 \pm 0.75$ & $1.67 \pm 0.83^{c}$ & $1.57 \pm 0.45$ & $1.72 \pm 0.52 \mathrm{a}$ \\
\hline
\end{tabular}

HDL-C, high-density lipoprotein cholesterol; LDL-C, low-density lipoprotein cholesterol. The quantitative variables were presented as mean \pm standard deviation and their difference between the groups was determined by the $t$-test. The values of triglyceride were presented as median (interquartile range), and their difference between the groups was determined by the Wilcoxon-Mann-Whitney test. The difference in percentage of cigarette smoking and alcohol consumption between the groups was determined by $x^{2}$-test.

a $P<0.001$ in comparison with males from the same ethnic group

b $P<0.01$ in comparison with males from the same ethnic group.

c $P<0.05$ in comparison with males from the same ethnic group.

\section{Results of genotyping}

After the genomic DNA of the samples was amplified by PCR, the purpose gene of $331 \mathrm{bp}$ nucleotide sequences could be seen in all samples (Figure 1A). The genotypes identified were labeled according to the presence or absence of the enzyme restriction sites. Thus, AA genotype is homozygote for the presence of the site (180- and 151-bp), AG genotype is heterozygote for the presence and absence of the site (331-, 180- and 151-bp) and GG genotype is homozygote for the absence of the site (331 bp; Figure 1B). The AA, AG and GG genotypes detected by PCR-RFLP were also confirmed by direct sequencing (Figure 2).

\section{Genotypic and allelic frequencies}

As shown in Table 2, the genotype and allele frequencies of ZNF259 rs2075290 SNP were different between the Mulao and Han populations $(P<0.001$ for each). The minor $\mathrm{G}$ allele frequency was higher in Mulao $(28.7 \%)$ than in Han $(23.3 \%)$. The genotype frequencies of rs2075290 SNP agreed with the Hardy-Weinberg equilibrium in both populations $(P>$
0.05 for each). Gender-subgroup analysis showed that the genotype frequencies of rs2075290 SNP between males and females were different in Han $(P<0.005)$ but not in Mulao.

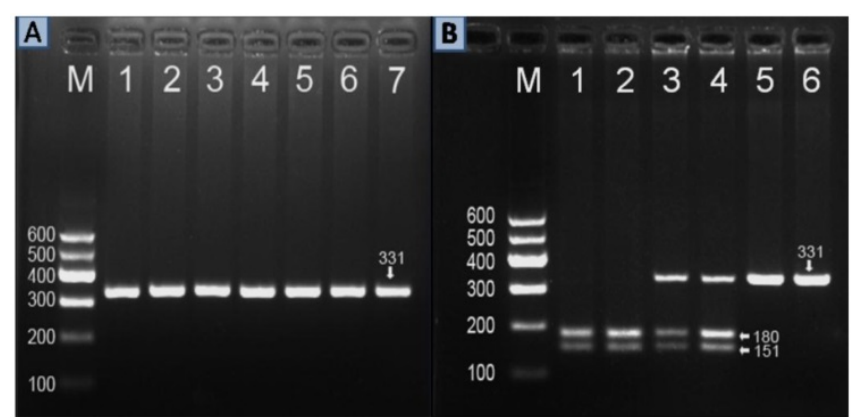

Figure I. Electrophoresis of PCR products and genotyping of the ZNF259 rs2075290 SNP. (A) Lane M, 100 bp marker ladder; lanes I-6, $33 \mathrm{I}$ bp band of PCR products. (B) Lane M, 100 bp marker ladder; lanes I and 2, AA genotype (I80- and I5I-bp); lanes 3 and 4, AG genotype (33I-, 180- and I5I-bp); and lanes 5 and 6, GG genotype (33I bp). 


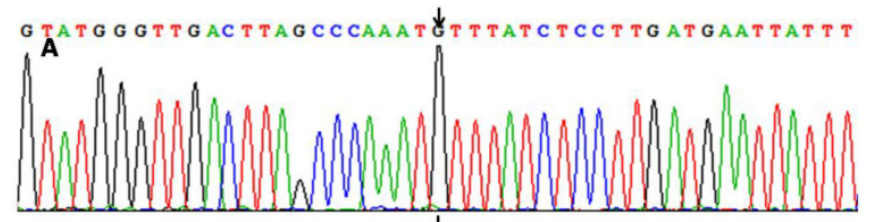

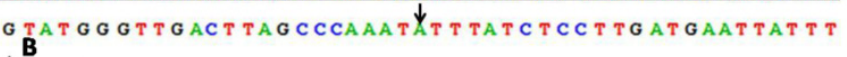

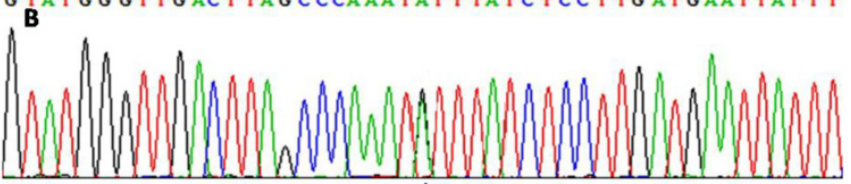

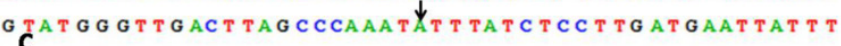

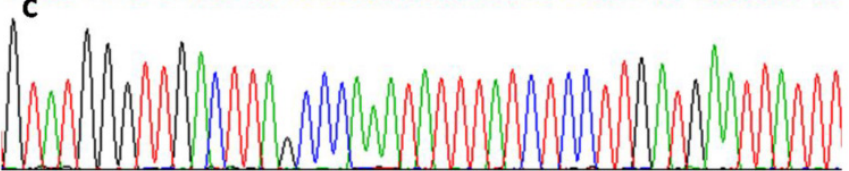

Figure 2. A part of the nucleotide sequences of the ZNF259 rs2075290 SNP by direct sequencing. (A) GG genotype; (B) AG genotype; (C) AA genotype.

\section{Genotypes and serum lipid levels}

Table 3 describes the association between genotypes and serum lipid levels. Serum levels of TG in Mulao males, and TC, TG and LDL-C in Mulao females were different between the AA and AG/GG genotypes $(P<0.05-0.001)$; the subjects with AG/GG genotypes had higher serum TC, TG and LDL-C levels than those with AA genotype. Serum TC, LDL-C and ApoA1 levels in Han males, and TG and ApoB levels and the ApoA1/ApoB ratio in Han females were different between the AA and AG/GG genotypes $(P<0.05-0.001)$; the AG/GG genotype subjects in Han males had lower TC, LDL-C and ApoA1 levels, but those in Han females had higher TG and ApoB levels and lower ApoA1/ApoB ratio than the AA genotype subjects.

Table 2. Comparison of the genotype and allele frequencies of the ZNF259 rs2075290 SNP between males and females of Mulao and Han populations.

\begin{tabular}{|c|c|c|c|c|c|c|c|c|c|}
\hline Genotype/Allele & Mulao & Han & $P$ value & Mulao/Male & Mulao/Female & $P$ value & Han/Male & Han/Female & $P$ value \\
\hline & $\mathrm{n}=788$ & $\mathrm{n}=778$ & & $\mathrm{n}=346$ & $\mathrm{n}=442$ & & $\mathrm{n}=307$ & $\mathrm{n}=471$ & \\
\hline $\mathrm{AA}[\mathrm{n}(\%)]$ & 395 (50.1) & $458(58.9)$ & & $174(50.3)$ & $221(50.0)$ & & $198(64.5)$ & $260(55.2)$ & \\
\hline AG [n (\%)] & $333(42.3)$ & $278(35.7)$ & & $151(43.6)$ & $182(41.2)$ & & $87(28.3)$ & $191(40.6)$ & \\
\hline GG [n (\%)] & $60(7.6)$ & $42(5.4)$ & 0.002 & $21(6.1)$ & $39(8.8)$ & 0.330 & $22(7.2)$ & $20(4.2)$ & 0.001 \\
\hline $\mathrm{A}[\mathrm{n}(\%)]$ & $1123(71.3)$ & 1194 (76.7) & & 499 (72.1) & 624 (70.6) & & $483(78.7)$ & $711(75.5)$ & \\
\hline $\mathrm{G}[\mathrm{n}(\%)]$ & $453(28.7)$ & $362(23.3)$ & 0.000 & $193(27.9)$ & $260(29.4)$ & 0.508 & $132(21.3)$ & $231(24.5)$ & 0.163 \\
\hline
\end{tabular}

Table 3. Comparison between the ZNF259 rs2075290 G>A genotypes and serum lipid levels.

\begin{tabular}{|c|c|c|c|c|c|c|c|c|}
\hline Genotype & $\mathrm{n}$ & TC & TG & HDL-C & LDL-C & ApoA1 & ApoB & ApoA1/ \\
\hline & & $(\mathrm{mmol} / \mathrm{L})$ & $(\mathrm{mmol} / \mathrm{L})$ & $(\mathrm{mmol} / \mathrm{L})$ & $(\mathrm{mmol} / \mathrm{L})$ & $(\mathrm{g} / \mathrm{L})$ & $(\mathrm{g} / \mathrm{L})$ & ApoB \\
\hline \multicolumn{9}{|c|}{ Mulao/Male } \\
\hline AA & 174 & $4.95 \pm 1.06$ & $1.05(0.91)$ & $1.71 \pm 0.42$ & $2.92 \pm 0.75$ & $1.33 \pm 0.39$ & $1.04 \pm 0.67$ & $1.56 \pm 0.71$ \\
\hline AG/GG & 172 & $5.03 \pm 1.09$ & $1.24(1.27)$ & $1.63 \pm 0.63$ & $2.94 \pm 0.86$ & $1.26 \pm 0.40$ & $1.02 \pm 0.66$ & $1.44 \pm 0.68$ \\
\hline$F$ & & 0.303 & -2.471 & 1.308 & 0.045 & 1.498 & 0.029 & 1.422 \\
\hline$P$ & & 0.583 & 0.013 & 0.254 & 0.832 & 0.222 & 0.866 & 0.234 \\
\hline \multicolumn{9}{|c|}{ Mulao/Female } \\
\hline AA & 221 & $4.76 \pm 1.21$ & $0.93(0.59)$ & $1.71 \pm 0.41$ & $2.82 \pm 0.90$ & $1.31 \pm 0.37$ & $0.90 \pm 0.47$ & $1.79 \pm 1.49$ \\
\hline AG/GG & 221 & $5.14 \pm 1.13$ & $1.07(0.68)$ & $1.78 \pm 0.41$ & $3.04 \pm 0.88$ & $1.31 \pm 0.42$ & $0.93 \pm 0.51$ & $1.66 \pm 0.72$ \\
\hline$F$ & & 9.024 & -3.554 & 1.261 & 5.390 & 0.000 & 0.280 & 0.987 \\
\hline$P$ & & 0.003 & 0.000 & 0.262 & 0.021 & 0.995 & 0.597 & 0.321 \\
\hline$F 1$ & & 2.086 & 0.795 & 1.555 & 0.386 & 2.425 & 0.551 & 0.161 \\
\hline$P 1$ & & 0.149 & 0.373 & 0.213 & 0.535 & 0.120 & 0.458 & 0.688 \\
\hline \multicolumn{9}{|l|}{ Han/Male } \\
\hline AA & 198 & $5.17 \pm 0.91$ & $1.14(0.92)$ & $1.71 \pm 0.41$ & $3.05 \pm 0.83$ & $1.38 \pm 0.29$ & $0.91 \pm 0.20$ & $1.63 \pm 0.47$ \\
\hline AG/GG & 109 & $4.86 \pm 0.89 \downarrow$ & $1.00(1.30)$ & $1.65 \pm 0.36$ & $2.81 \pm 0.63 \downarrow$ & $1.25 \pm 0.21 \downarrow$ & $0.89 \pm 0.16$ & $1.55 \pm 0.35$ \\
\hline$F$ & & 7.763 & -0.787 & 1.058 & 6.216 & 5.249 & 0.750 & 1.963 \\
\hline$P$ & & 0.006 & 0.431 & 0.305 & 0.013 & 0.023 & 0.387 & 0.162 \\
\hline \multicolumn{9}{|c|}{ Han/Female } \\
\hline AA & 260 & $4.70 \pm 0.98$ & $0.88(0.67)$ & $1.80 \pm 0.69$ & $2.75 \pm 0.82$ & $1.31 \pm 0.23$ & $0.79 \pm 0.18$ & $1.80 \pm 0.33$ \\
\hline AG/GG & 211 & $4.90 \pm 1.12$ & $1.07(0.78)$ & $1.75 \pm 0.40$ & $2.92 \pm 0.95$ & $1.33 \pm 0.26$ & $0.84 \pm 0.20$ & $1.68 \pm 0.47$ \\
\hline F & & 3.734 & -3.985 & 0.406 & 2.409 & 0.523 & 5.112 & 5.043 \\
\hline $\mathrm{P}$ & & 0.054 & 0.000 & 0.524 & 0.121 & 0.470 & 0.024 & 0.025 \\
\hline$F 1$ & & 10.571 & 0.926 & 0.052 & 5.828 & 4.490 & 2.957 & 0.145 \\
\hline P1 & & 0.001 & 0.336 & 0.820 & 0.016 & 0.034 & 0.086 & 0.703 \\
\hline
\end{tabular}

TC, total cholesterol; TG, triglyceride; HDL-C, high-density lipoprotein cholesterol; LDL-C, low-density lipoprotein cholesterol; ApoA1, apolipoprotein A1; ApoB, apolipoprotein $B ; A$ poA1/ApoB, the ratio of apolipoprotein A1 to apolipoprotein B. The values of triglyceride were presented as median (interquartile range), and their difference among the genotypes was determined by the Kruskal-Wallis test or the Wilcoxon-Mann-Whitney test. The values of $F 1$ and P1 are the interaction between ZNF259 rs2075290 SNP and gender on serum lipid levels. 
Table 4. Correlation between the genotypes/alleles of the ZNF259 rs2075290 SNP and serum lipid levels in the Mulao and Han populations.

\begin{tabular}{|c|c|c|c|c|c|c|}
\hline Lipid & Genotype/allele & Unstandardized coefficient & Standard error & Standardized coefficient & $t$ & $P$ \\
\hline \multicolumn{7}{|l|}{ Mulao plus Han } \\
\hline TG & Genotype & 0.222 & 0.043 & 0.131 & 5.178 & 0.000 \\
\hline ApoA1/ApoB & Allele & -0.099 & 0.034 & -0.075 & -2.936 & 0.003 \\
\hline \multicolumn{7}{|l|}{ Mulao } \\
\hline $\mathrm{TC}$ & Genotype & 0.182 & 0.064 & 0.103 & 2.838 & 0.005 \\
\hline TG & Genotype & 0.247 & 0.058 & 0.152 & 4.231 & 0.000 \\
\hline LDL-C & Genotype & 0.108 & 0.049 & 0.081 & 2.226 & 0.026 \\
\hline \multicolumn{7}{|l|}{ Mulao/Male } \\
\hline TG & Allele & 0.359 & 0.137 & 0.143 & 2.626 & 0.009 \\
\hline \multicolumn{7}{|l|}{ Han } \\
\hline \multirow[t]{2}{*}{ ApoB } & Allele & -0.176 & 0.048 & -0.440 & -3.641 & 0.000 \\
\hline & Genotype & 0.121 & 0.039 & 0.367 & 3.084 & 0.002 \\
\hline \multicolumn{7}{|l|}{ Han/Male } \\
\hline TC & Allele & -1.015 & 0.278 & -0.542 & -3.650 & 0.000 \\
\hline TG & Genotype & 0.246 & 0.116 & 0.121 & 2.117 & 0.035 \\
\hline HDL-C & Genotype & -0.119 & 0.038 & -0.177 & -3.121 & 0.002 \\
\hline LDL-C & Allele & -0.563 & 0.125 & -0.358 & -4.517 & 0.000 \\
\hline ApoA1 & Allele & -0.103 & 0.033 & -0.178 & -3.148 & 0.002 \\
\hline АроB & Allele & -0.196 & 0.064 & -0.490 & -3.061 & 0.002 \\
\hline \multicolumn{7}{|l|}{ Han/Female } \\
\hline TC & Genotype & 0.167 & 0.083 & 0.093 & 2.010 & 0.044 \\
\hline
\end{tabular}

TC, total cholesterol; TG, triglyceride; HDL-C, high-density lipoprotein cholesterol; LDL-C, low-density lipoprotein cholesterol; ApoA1, apolipoprotein A1; ApoB, apolipoprotein B.

\section{Interactions between genotypes and gender on serum lipid levels}

An interaction between ZNF259 rs2075290 polymorphism and male gender on serum TC, LDL-C, and ApoA1 levels was noted in Han population $(P<$ 0.05-0.01; Table 3) but not in Mulao's. The subjects with AG/AA genotype in Han males had lower TC, LDL-C and ApoA1 levels compared to the individuals with GG genotype. On the other hand, the AG/AA genotype individuals in Han females had higher TG and ApoB levels compared to GG genotype individuals.

\section{Relative factors for serum lipid parameters}

Multiple linear regression analyses showed that the levels of TC, TG and LDL-C in Mulao, and ApoB in Han were correlated with genotypes $(P<$ 0.05-0.001; Table 4). In gender subgroups, the levels of TG in Mulao males, TC, TG, HDL-C, LDL-C, ApoA1 and $\mathrm{ApoB}$ in Han males and TC in Han females were correlated with genotypes $(P<0.05-0.001$; Table 4$)$. Several environmental factors such as age, weight, waist circumference, alcohol consumption and cigarette smoking, and traditional cardiovascular risk factors such as BMI, fasting blood glucose and blood pressure levels were also correlated with serum lipid parameters in males and females of both ethnic groups (Supplementary Table 1).

\section{Discussion}

In the current study, we showed that serum lipid profiles were significantly different between males and females in both Mulao and Han ethnic groups. As expected, the males had higher serum levels of bad cholesterols and lower levels of good cholesterols than the females in both ethnic groups. A significant difference in the genotype and allele frequencies of ZNF259 rs2075290 SNP was also noted between the two ethnic populations. The minor $G$ allele frequencies in Mulao and Han were $28.7 \%$ and $23.3 \%$ respectively, which were in close proximity to those of Chinese Han Bejing (25.6\%) reported in international haplotype map (HapMap) project. On gender subgroup analysis, the genotype frequencies between males and females were different in Han but not in Mulao. According to HapMap data, the minor allele frequency of rs2075290 was $25.5 \%$ in Japanese, and $4.0 \%$ in Inbadan Yoruba and Utahns. Apparently, the minor allele frequency was higher in Asian than the Western populations. These findings suggest that genotype and allele frequencies of ZNF259 rs2075290 SNP are inconsistent among diverse ethnic groups or between males and females.

A recent meta-analysis including 13 independent studies reported that ZNF259 rs2075290 SNP was the top association SNP with MetS in Eurpean ancestry [13]. The minor allele was significantly associated with pleiotropic (one SNP influence many traits) ef- 
fects on increased HDL-C and TG, and waist circumference and TG [13]. In the present study, the ZNF259 rs2075290 SNP was correlated with TG in the combined Mulao and Han population, TC, TG and LDL-C in Mulao, and ApoB in Han. However, no association with HDL-C was detected either in Mulao, Han or the combined population. The reason for this discrepancy is not fully understood. It might be due to the differences in genetic backgrounds, dietary habits, and environmental factors between the two ethnic populations and/or simply due to the low power of this study. It is well accepted that ethnic differences in serum lipid levels were partly due to the differences in the dietary intakes [21]. Diet alone could account for up to $2.5 \%$ of the variability on serum lipid levels [22-26]. Although rice and corn are the staple foods for both ethnic groups; Mulao peoples have a typical habit of eating cold foods along with acidic and spicy dishes, local bean soy sauce, pickled vegetables and animal offal's which contain abundant saturated fatty acid. Therefore, it is possible that the difference in dietary habit between Mulao and Han ethnic groups partly contribute variability in the effect of ZNF259 rs2075290 SNP on serum lipid levels.

Several SNPs in ZNF259 were reported to have sex specific effects on serum lipid levels. Xu et al. reported that the minor allele of ZNF259 rs964184 was a risk allele for CAD in Han females (odds ratio: 1.49, 95\% confidence interval: $1.00-2.22, P=0.05$ ) but not in males [27]. A sex-stratified meta-analysis of 49 GWAS identified lipid loci also showed that the minor $\mathrm{C}$ allele of rs28927680 and rs3135506 SNPs near ZNF259 region exhibited sex (male) - specific association of increased TG in European Americans and Mexican Americans/Hispanics; but the association was failed to replicate in American Indians and African Americans [12]. Here, we found that the minor $G$ allele of ZNF259 rs2075290 SNP was associated with higher serum TC levels in Mulao males, higher TC, TG and LDL-C levels in Mulao females. On the other hand, the minor $G$ allele was associated with lower TC, LDL-C and ApoA1 levels in Han males, and higher TG and ApoB levels and lower ApoA1/ApoB ratio in Han females. Therefore, the minor allele carriers in Han men have more favorable lipid profiles (lower TC and LDL-C) than the minor allele non-carriers. To the best of our knowledge, this study is the first attempt to report the gender specific association of ZNF259 rs2075290 SNP. Therefore, further studies with larger sample size are still needed to confirm this association.

Several environmental factors were also correlated with serum lipid levels in males and females of both Mulao and Han populations. In the current study, the males had significantly higher values of weight, height, BMI, waist circumference compared to the female counterparts in both ethnic groups. Garcia-Palmieri et al. stated that diet and relative weight could account for up to $6 \%$ of the variability in serum cholesterol levels [22]. In particular, for every $1-\mathrm{kg}$ decrease in body weight, TG decreased by 0.011 $\mathrm{mmol} / \mathrm{L}$ and HDL-C increased by $0.011 \mathrm{mmol} / \mathrm{L}$ [28]. In addition, in this study, the males' percentages of subjects consuming alcohol and cigarette smoking were significantly higher than the females' in both ethnic groups. Rimm et al. documented that consuming of $30 \mathrm{~g}$ of ethanol per day increased the concentrations of HDL-C by $3.99 \mathrm{mg} / \mathrm{dl}$, ApoA1 by 8.82 $\mathrm{mg} / \mathrm{dl}$, and TG by $5.69 \mathrm{mg} / \mathrm{dl}$ [29]. Yin et al. also showed that BMI, cigarette smoking and alcohol consumption could interact with certain lipid-related gene variants to modify the serum lipid levels in Bai $\mathrm{Ku}$ Yao and Han Chinese ethnic groups [30, 31]. Therefore, the results of exposure to different environmental factors may further modify the effect of genetic variation on serum lipid levels in our study populations.

There are some potential limitations in our study. First, it is undeniable that this study has insufficient power to produce a robust conclusion; therefore, such a small-scale study needs to replicate in independent cohorts. Second, the cross-sectional study design limits the ability to determine any causality of the relationships observed. Third, the impact of diet was not evaluated in this study. It is possible that part of the relationship observed in this study may be partly influenced by the effect of dietary intake.

\section{Conclusion}

An interaction between ZNF259 rs2075290 polymorphism and male gender on serum TC, LDL-C, and ApoA1 levels is noted in Han population $(P<$ 0.05-0.01) but not in Mulao's. The subjects with AG/GG genotype in Mulao males (higher TG), Mulao females (higher TC, TG and LDL-C) and Han females (higher TG and ApoB) have less favorable lipid profiles than those with AA genotype. In contrast, the subjects with AG/GG genotype in Han men have more favorable lipid profiles (lower TC and LDL-C) than those with AA genotype. These findings suggest that the association between ZNF259 rs2075290 SNP and serum lipid levels might have ethnic- and/or sex-specificity.

\section{Supplementary Material}

Supplementary Table 1.

http://www.medsci.org/v11p0471s1.pdf 


\section{Acknowledgements}

This study was supported by the National Natural Science Foundation of China (No: 30960130).

\section{Competing Interests}

The authors have declared that no competing interest exists.

\section{References}

1. Mittendorfer B. Sexual dimorphism in human lipid metabolism. J Nutr. 2005;135(4):681-686.

2. Wang X, Magkos F, Mittendorfer B. Sex differences in lipid and lipoprotein metabolism: it's not just about sex hormones. J Clin Endocrinol Metab. 2011;96(4):885-893

3. Cullen P. Evidence that triglycerides are an independent coronary heart disease risk factor. Am J Cardiol. 2000;86(9):943-949.

4. Meagher EA. Addressing cardiovascular disease in women: focus on dyslipidemia. J Am Board Fam Pract. 2004;17(6):424-437.

5. Teo YY, Sim X. Patterns of linkage disequilibrium in different populations: implications and opportunities for lipid-associated loci identified from genome-wide association studies. Curr Opin Lipidol. 2010;21(2):104-115.

6. Teslovich TM, Musunuru K, Smith AV, et al. Biological, clinical and population relevance of 95 loci for blood lipids. Nature. 2010;466(7307):707-713.

7. Saleheen D, Soranzo N, Rasheed A, et al. Genetic determinants of major blood lipids in Pakistanis compared with Europeans. Circ Cardiovasc Genet. 2010;3(4):348-357.

8. Schunkert H, Konig IR, Kathiresan S, et al. Large-scale association analysis identifies 13 new susceptibility loci for coronary artery disease. Nat Genet. 2011;43(4):333-338

9. Braun TR, Been LF, Singhal A, et al. A replication study of GWAS-derived lipid genes in Asian Indians: the chromosomal region 11q23.3 harbors loci contributing to triglycerides. PLoS One. 2012;7(5):e37056.

10. Galcheva-Gargova Z, Gangwani L, Konstantinov KN, et al. The cytoplasmic zinc finger protein ZPR1 accumulates in the nucleolus of proliferating cells. Mol Biol Cell. 1998;9(10):2963-2971.

11. Gangwani L, Mikrut M, Theroux S, et al. Spinal muscular atrophy disrupts the interaction of ZPR1 with the SMN protein. Nat Cell Biol. 2001;3(4):376-383.

12. Taylor KC, Carty CL, Dumitrescu L, et al. Investigation of gene-by-sex interactions for lipid traits in diverse populations from the population architecture using genomics and epidemiology study. BMC Genet. 2013;14:33.

13. Kraja AT, Vaidya D, Pankow JS, et al. A bivariate genome-wide approach to metabolic syndrome: STAMPEED consortium. Diabetes. 2011;60(4):1329-1339.

14. $\mathrm{Xu} \mathrm{L}$, Deng QY, Li SF, et al. Genetic analysis of Mulao nationality using 15 short tandem repeats. Zhonghua $Y_{i}$ Xue $Y_{i}$ Chuan Xue Za Zhi. 2008;25(1):96-100.

15. Aung LH, Yin RX, Wu DF, et al. Association of the TRIB1 tribbles homolog 1 gene rs17321515 $\mathrm{A}>\mathrm{G}$ polymorphism and serum lipid levels in the Mulao and Han populations. Lipids Health Dis. 2011;10:230.

16. Aung LH, Yin RX, Wu DF, et al. Association of the apolipoprotein M gene polymorphisms and serum lipid levels. Mol Biol Rep. 2013;40(2):1843-1853.

17. Aung LH, Yin RX, Miao L, et al. The proprotein convertase subtilisin/kexin type 9 gene E670G polymorphism and serum lipid levels in the Guangxi Bai $\mathrm{Ku}$ Yao and Han populations. Lipids Health Dis. 2011;10:5.

18. Ruixing Y, Qiming F, Dezhai Y, et al. Comparison of demography, diet, lifestyle, and serum lipid levels between the Guangxi Bai Ku Yao and Han populations. J Lipid Res. 2007;48(12):2673-2681.

19. Ruixing Y, Dezhai $Y$, Shuquan L, et al. Hyperlipidaemia and its risk factors in the Guangxi Bai $\mathrm{Ku}$ Yao and Han populations. Public Health Nutr. 2009;12(6):816-824

20. Aung LH, Yin RX, Wu DF, et al. Proprotein convertase subtilisin/kexin type 9 gene E670G polymorphism interacts with alcohol consumption to modulate serum lipid levels. Int J Med Sci. 2013;10(2):124-132.

21. Bermudez OI, Velez-Carrasco W, Schaefer EJ, et al. Dietary and plasma lipid, lipoprotein, and apolipoprotein profiles among elderly Hispanics and non-Hispanics and their association with diabetes. Am J Clin Nutr. 2002;76(6):1214-1221

22. Garcia-Palmieri MR, Tillotson J, Cordero $\mathrm{E}$, et al. Nutrient intake and serum lipids in urban and rural Puerto Rican men. Am J Clin Nutr. 1977;30(12):2092-2100.

23. Sola R, Fito M, Estruch R, et al. Effect of a traditional Mediterranean diet on apolipoproteins B, A-I, and their ratio: a randomized, controlled trial. Atherosclerosis. 2011;218(1):174-180.

24. Valente EA, Sheehy ME, Avila JJ, et al. The effect of the addition of resistance training to a dietary education intervention on apolipoproteins and diet quality in overweight and obese older adults. Clin Interv Aging. 2011;6:235-241.

25. Watts GF, Jackson P, Burke V, Lewis B. Dietary fatty acids and progression of coronary artery disease in men. Am J Clin Nutr. 1996;64(2):202-209.

26. Grundy SM, Denke MA. Dietary influences on serum lipids and lipoproteins. J Lipid Res. 1990;31(7):1149-1172.
27. Xu L, Zhou J, Huang S, et al. An association study between genetic polymorphisms related to lipoprotein-associated phospholipase $\mathrm{A}(2)$ and coronary heart disease. Exp Ther Med. 2013;5(3):742-750.

28. Yu-Poth S, Zhao G, Etherton T, et al. Effects of the National Cholesterol Education Program's Step I and Step II dietary intervention programs on cardiovascular disease risk factors: a meta-analysis. Am J Clin Nutr. 1999;69(4):632-646

29. Rimm EB, Williams P, Fosher K, et al. Moderate alcohol intake and lower risk of coronary heart disease: meta-analysis of effects on lipids and haemostatic factors. BMJ. 1999;319(7224):1523-1528

30. Yin RX, Wu DF, Miao L, et al. Interactions of several single nucleotide polymorphisms and high body mass index on serum lipid traits. Biofactors. 2013;28(10).

31. Yin $\mathrm{RX}, \mathrm{Wu} \mathrm{DF}, \mathrm{Miao} \mathrm{L}$, et al. Several genetic polymorphisms interact with overweight/obesity to influence serum lipid levels. Cardiovasc Diabetol. 2012;11:123 